Yu Wei, et al., Int. J. of Safety and Security Eng., Vol. 6, No. 2 (2016) 238-245

\title{
COMPARING ALTERNATIVE BUDGET ALLOCATION MODELS TO SUPPORT STRATEGIC WILDLAND FIRE PROGRAM ANALYSIS ACROSS US NATIONAL PARKS
}

\author{
YU WEI ${ }^{1}$, DOUGLAS RIDEOUT ${ }^{1}$, ANDY KIRSCH $^{2} \&$ NIKI KERNOHAN ${ }^{1}$ \\ ${ }^{1}$ Colorado State University, USA. \\ ${ }^{2}$ National Park Service, USA.
}

\begin{abstract}
Hazard fuel reduction and wildland fire preparedness programs are two important budgeting components in the US National Park Service strategic wildland fire planning. During the planning process, each national park independently conducts analysis to understand the benefits from investing in each program to mitigate fire risks and improve ecosystem benefits. The national program analysis imports the cost-effective frontiers of investment in both programs from each national park. The national program then allocates cost-effective funding to the parks and implements required national policies while minimizing disruption to current programs of work. In this study, we test and compare two alternative modeling methods for budget allocation between the fuel treatment and preparedness programs responding to changes in funding levels nationally. One approach uses a nonlinear programming model (NLP) to maximize the benefits of investments in both programs with a set of feasibility constraints. The other approach uses a simulation-based gradient method to manage program budget changes. Both approaches are designed to focus on national level program efficiency while mitigating potential program disruptions; however, different approaches suggest different budgeting allocation strategies. This study compares the trade-offs between efficiency and the level of disruption of different budget allocation methods. Discoveries could help managers to select and implement an efficient and viable analytical system to study the value of funding increases, the cost of budget reductions, and guide landscape allocations. It will also identify national impacts by accumulating allocations to individual units across the national parks in the United States.

Keywords: fuel treatment, gradient method, linear programming, preparedness.
\end{abstract}

\section{INTRODUCTION}

Wildfire is a natural component of many terrestrial ecosystems with the potential to threaten human lives or destroy property and natural resources [1]. Attempts to control fire risk usually focus on hazard fuel reduction/management or fire suppression [2]. These two important elements in wildland fire management are also strongly inter-related with one another [3].

The importance of fuel treatment programs is reflected in the federal budget and in the legislation. The President's 2015 draft budget emphasizes the importance of fuel treatments to create resilient landscapes [4]. Maintenance treatments are also highlighted as a means of keeping previously treated areas from degrading. Cost-effective fuel treatment programs appear consistently as a federal priority in both the FLAME Act [5] and the Healthy Forest Restoration Act [6].

Initial attack (IA) is one of the most effective fire suppression actions. It involves the actions taken by the first resources to arrive at a wildfire or wildland fire incident [7]. 
Resources needed to respond to IA depending upon fire danger, fuel type, and values to be protected. The success of IA often depends on the investment made in the preparedness program in a land management unit. Fire authorities must look ahead and make preparedness planning decisions. Investment in preparedness improves IA success rate. These decisions include determining what type and amount of suppression resources need to be acquired and where to base these resources to best satisfy demand [8].

This research introduces a method that can be used to synergize park level fuel treatment and preparedness analysis results to support the budget allocation decisions taken for the national level fire program. The outputs from this research can provide managers with quantitative data that can be used to support, defend, and communicate their management decisions.

\section{METHODS}

We introduce two modeling methods for fuel treatment and preparedness budget allocation between multiple national parks (or other management units) across the United States. The first method uses a nonlinear programming model (NLP) to maximize the total investment return under various budget levels. This approach provides the most efficient budget allocation strategies under a set of preselected budgeting constraints. The second method uses a gradient-based approach to gradually change the budget allocations between the fuel treatment and preparedness programs across multiple national parks. This approach is designed specifically to avoid a potentially dramatic budget change that may be disruptive to the fire management program. We describe these two methods in this section and implement them in a budget reallocation study across thirteen national parks in the United States.

2.1 Develop response surface to track the joint benefits from the fuel treatment and IA preparedness programs

We use $p$ to denote the preparedness program and $f$ for the fuel treatment program; each national park is indexed by $i$. For each park $i$, at a given preparedness budget $\left(B_{p, i}\right)$ and a given fuel treatment budget $\left(B_{f, i}\right)$, the total benefit $V_{i}$ from the fire program can be calculated by the function:

$$
V_{i}=q\left(B_{p, i}, B_{f, i}\right)
$$

The total fire program budget for park $i$ is calculated as:

$$
B_{i}=B_{p, i}+B_{f, i}
$$

Equation (1) takes a different form for each specific national park. In this study, we use the empirical fuel treatment and preparedness analyses in each park [9] to fit a set of nonlinear equations to create the response surface of $q\left(B_{p, i}, B_{f, i}\right)$ for each park $i$.

For example, in Sequoia and Kings Canyon National Parks (SEKI), eqn (1) is specified as below with coefficients $\beta_{0}=1273, \beta_{1}=83.1, \beta_{2}=-964$, and $\beta_{3}=0.748$ :

$$
V_{S E K I}=\beta_{0}+\beta_{1}\left(B_{p, S E K I}\right)+B_{f, S E K I}+\left(\frac{\beta_{2}}{\beta_{3}+B_{p, S E K I}+B_{p, S E K I} B_{f, S E K I}+\sqrt{B_{f, S E K I}}}\right)
$$

For practical purpose, in this study, we focus on using these contiguous functions to calculate the total investment return at all budget levels within a certain range from both the preparedness and the fuel treatment programs in a park (such as SEKI). The nonlinear function produces a smooth contiguous surface. The coefficient $\beta_{0}, \beta_{1}, \beta_{2}$ and $\beta_{3}$ may not have a direct economic interpretation. These nonlinear equations are convex and differentiable 


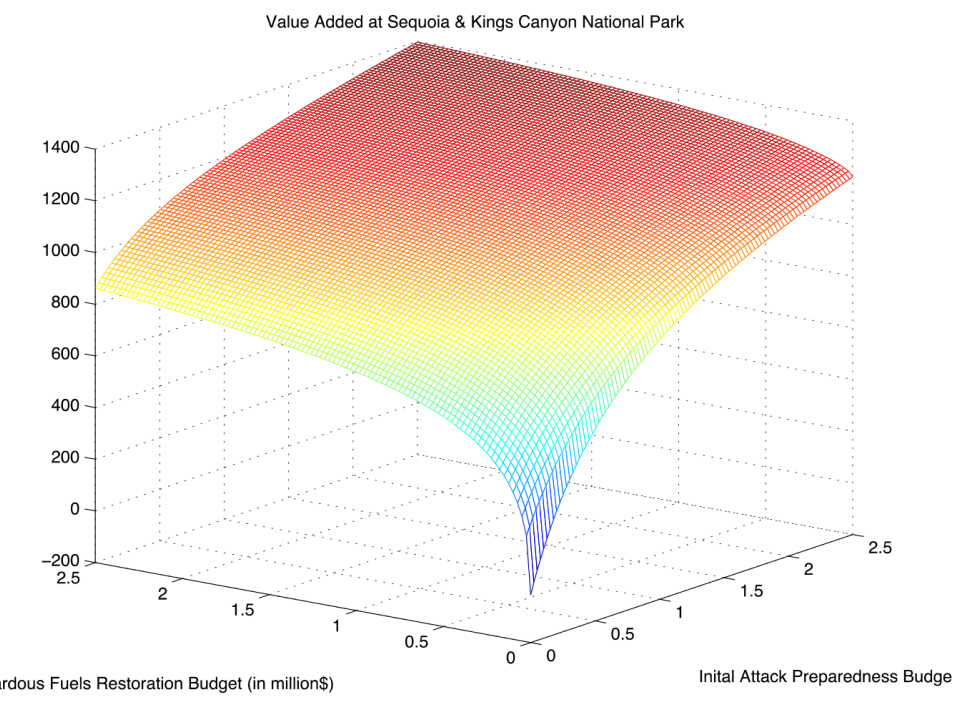

Error metrics:

$\begin{array}{ll}\text { Mean Absolute Error } & 25.5961 \\ \text { Mean Square Error } & 950.474 \\ \mathrm{R}^{2} \text { Goodness of Fit } & 0.989758 \\ \text { Correlation Coefficient } & 0.994866 \\ \text { Rank Correlation } & 0.988385 \\ \text { Maximum Error } & 66.7671 \\ \text { Logarithmic Error } & 5.9422\end{array}$

Figure 1: A contiguous surface and corresponding mathematical function are used to approximate the value added function (investment return) from different combinations of IA preparedness and hazardous fuels reduction budgets at SEKI. Error metrics show the accuracy of the approximation. Given a budget level, this surface can be used to determine the optimal combination of investment in the IA preparedness and the fuel reduction programs to maximize the total investment return. The graph is constructed based on empirical data from park level analysis [9].

within a reasonable budget range so that they can be used to support those nonlinear programming models or the gradient method to search for an optimal budget allocation across parks. An example of this type of nonlinear equations for SEKI is demonstrated in Fig. 1 with the corresponding error matrix.

\subsection{Budget allocation in each park using a NLP}

We first need to explore the optimal investment strategies under different fire program budgets in each national park. One approach is to use the set of nonlinear functions (i.e. eqn (1)) fitted from the empirical data from each park to develop a NLP for each park to optimally split the total park fire program budget into the fuel treatment and preparedness programs, 
potentially with additional constraints (discussed later) to limit the disruption of current budget structure within each park.

$\operatorname{Max} \quad \boldsymbol{q}\left(\boldsymbol{B}_{p, i}, \boldsymbol{B}_{f, i}\right)$

Subject to

$$
\begin{aligned}
& \left(\boldsymbol{B}_{p, i}+\boldsymbol{B}_{f, i}\right) \leq \mathbf{B}_{i} \\
& \boldsymbol{B}_{p, i}, \boldsymbol{B}_{f, i} \geq \mathbf{0}
\end{aligned}
$$

Decision variables in the above NLP models are the budget $B_{i}$ to be allocated separately to the hazard fuel reduction program and the preparedness program in each park $i$. The objective function of eqn (3) of this NLP model maximizes the total investment return in park $i$. To explore the benefit of increasing the overall fire program budget in park $i$, we can systematically increase the right hand side of eqn (4) and rerun this NLP model repeatedly.

The above NLP model can be further constrained with additional equations. For example, by adding eqn (6), we ensure that if the total fire program budget $B_{i}$ in park $i$ increased, neither the fuel program budget nor the preparedness program budget in that park should decrease.

$$
\begin{gathered}
\boldsymbol{B}_{p, i} \geq \boldsymbol{B}_{p, i}^{0} \\
\boldsymbol{B}_{f, i} \geq \boldsymbol{B}_{f, i}^{0}
\end{gathered}
$$

$\boldsymbol{B}^{0}{ }_{p, i}$ and $\boldsymbol{B}^{0}{ }_{f, i}$ each denote the IA preparedness and fuel treatment budget before the total fire program budget in park $i$ is increased and $\boldsymbol{B}_{p, i}$ and $\boldsymbol{B}_{f, i}$ are the new budgets for each program after the total budget increased to $\boldsymbol{B}_{\boldsymbol{i}}$. We name this NLP model with constraint (6) and (7) the 'NLP with nondeclining program budget' (NLP with NDPB). This NLP with NDPB model is designed to keep the balance between investment efficiency and budget stability in each park.

\subsection{Budget allocation using gradient method for individual parks}

A gradient method can also be used to address the budget allocation problem between the fuel treatment and preparedness programs in each park. We again will use objective function $\boldsymbol{q}\left(\boldsymbol{B}_{p, i}, \boldsymbol{B}_{f, i}\right)$ to support the search for optimal budget allocation. When the fire program budget is increased, we will search for a new budget allocation between the fuel treatment and preparedness programs with the search direction defined as the gradient of the objective function at the point defined by the current fire program budget allocation.

Figure 2 illustrates the differences between the NLP model with the NDPB method and the gradient-based method. The gradient method increases the budget along the direction of the highest investment return from the current budget level (local optimal) and may also require the least disruption to the current fire program budget in each individual park because it not only prevents the budget from decreasing in both programs, but it also keeps the rate of the budget increasing proportionally to the benefit of the budget increase in the fuel and the preparedness programs in each park.

\subsection{Budget allocation at national level}

It is straightforward to transfer the NLP model into a national level budgeting model to allocate the budget to different parks. That national model may take the form as described below. 


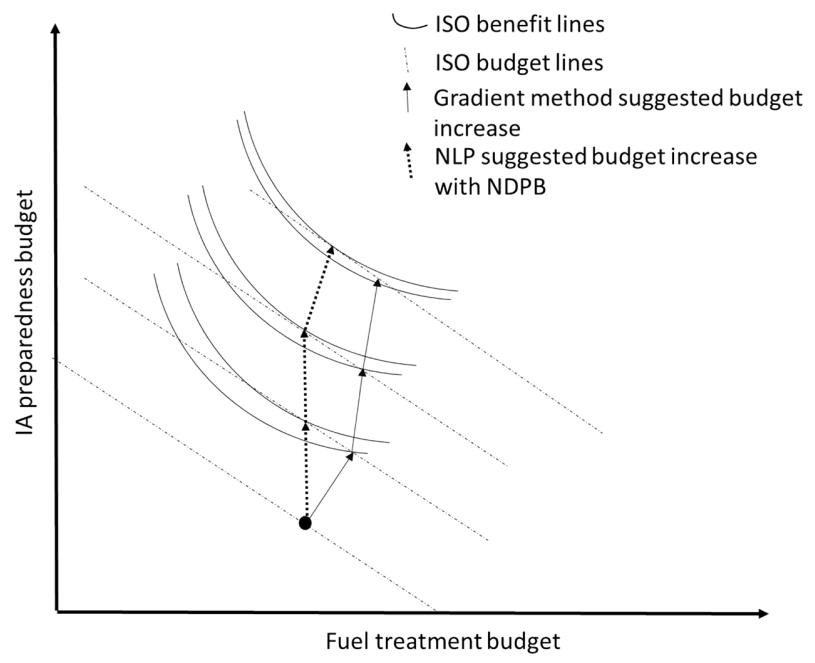

Figure 2: A diagram demonstrates the searching paths from the NLP model with NDPB, and from the gradient method.

$$
\operatorname{Max} \quad \sum_{i} \boldsymbol{q}\left(\boldsymbol{B}_{p, i}, \boldsymbol{B}_{f, i}\right)
$$

\section{Subject to}

$$
\begin{aligned}
& \sum_{i}\left(\boldsymbol{B}_{p, i}+\boldsymbol{B}_{f, i}\right) \leq \boldsymbol{B} \\
& \boldsymbol{B}_{p, i} \geq \boldsymbol{B}_{p, i}^{\mathbf{0}} \quad \forall \mathbf{i} \\
& \boldsymbol{B}_{f, i} \geq \boldsymbol{B}_{f, i}^{\mathbf{0}} \quad \forall \mathbf{i} \\
& \boldsymbol{B}_{p, i}, \boldsymbol{B}_{f, i} \geq \mathbf{0} \quad \forall \mathbf{i}
\end{aligned}
$$

However, this single-step approach cannot be applied easily for the gradient-based method. To implement the gradient method for national level budget reallocation and compare it with the NLP with NDPB method, we need to first study the step-by-step effect from budget increase in individual parks before scale the information up to the national level. This process has the following steps:

1. Calculate the marginal return from each additional amount of investment from the current budget level as well as the corresponding investment return in each park.

2. Increase the national level budget by $x$ dollar (a small amount); allocate this new investment to the park that has the highest marginal investment return; stop if all investment dollars are used up.

3. Go back to step 1) to update the marginal investment return from the individual park that has received the $x$ dollars. 


\section{RESULTS}

3.1 Budget allocation at individual parks

As mentioned, using the NLP model directly could introduce unacceptable disruptions and instability to the current fire programs. A less disruptive approach would be to use the NLP model with NDPB constraints eqns (6) and (7). Another method is to use the gradient-based approach. In this study, we compare only those results between the NLP model with NDPB and the gradients method. Both approaches are potentially more practical in the real world application for budget management. Figure 3a shows the comparison of investment returns of these two methods at SEKI; Figure 3b demonstrates the comparison in the Grand Teton

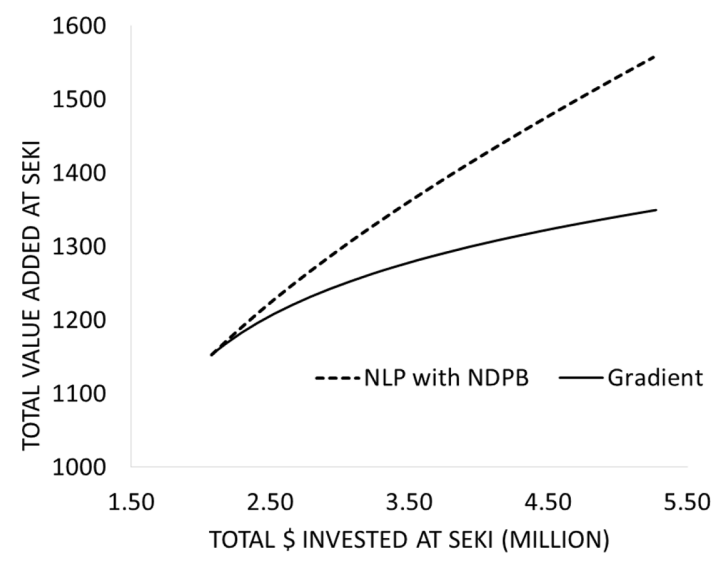

Figure 3a: Comparison of investment returns between the NLP with NDPB method and the gradient method for SEKI.

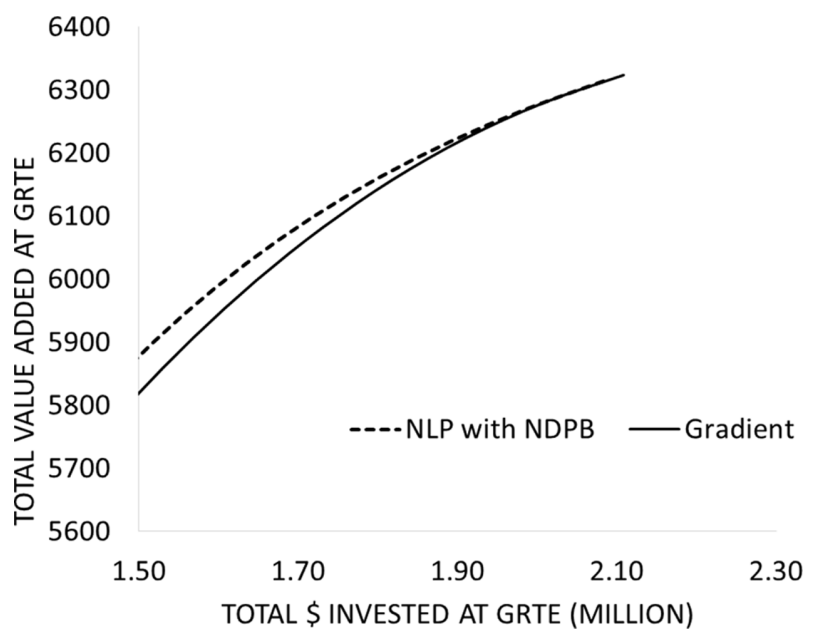

Figure 3b: Comparison of investment returns between the NLP with NDPB method and the gradient method for GRTE. 


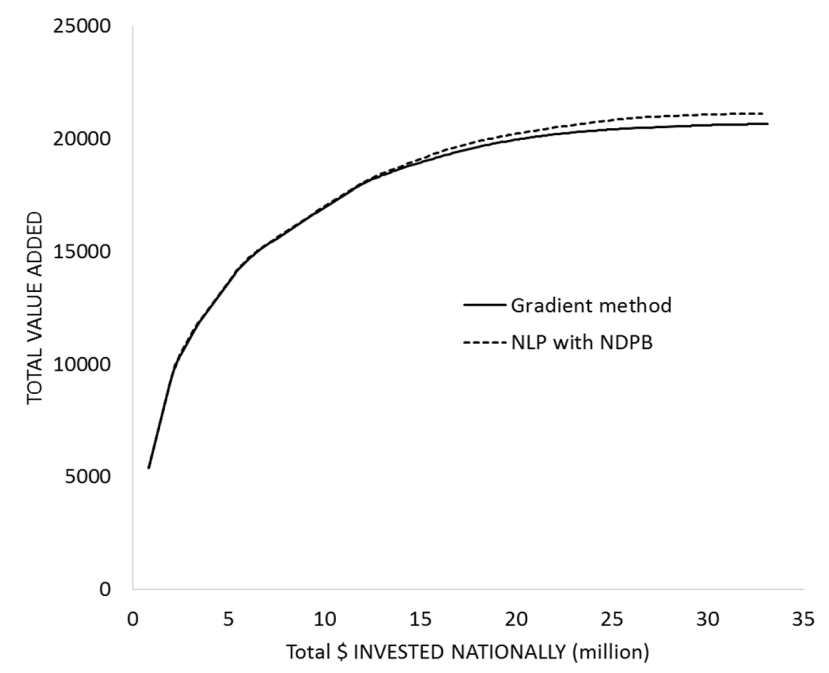

Figure 4: Comparison of the total investment return across the 13 national parks in the United States at different budget levels between using the NLP with NDPB method and using the gradient-based methods.

National Park (GRTE). From an investment efficiency aspect, the NLP model with NDPB constraints performs better than the gradient approach, but the difference is not substantial in GRTE. A similar trend holds for most of the 13 national parks tested.

\subsection{Budget allocation at the national level}

Budget allocation analysis for each park solves the problem of how to efficiently distribute a park-specific fuel treatment budget and IA preparedness budget without excessively disrupting the existing park level fire program. National level budget analysis uses the results generated from the analysis of each of the 13 parks to allocate fuel treatment and IA preparedness budgets to each park across the system. To ensure efficiency, any additional budget that has the potential to produce the highest marginal return on investment will be first invested in the fuel or preparedness program of the park. A preliminary study from the national analysis is demonstrated in Figure 4 based on the results from individual park analyses using either the NLP with NDPB model or the gradient-based searching approach. Overall, the NLP with NDPB approach is slightly more efficient in investment return; however, at the national level this difference is not substantial.

\section{CONCLUSIONS AND DISCUSSIONS}

This study introduces and compares two modeling methods to allocate budget in the fuel treatment program and the IA preparedness program across 13 national parks across the United States. The NLP with NDPB method maximizes the investment return in both programs at the park level and the national level with hardcoded constraints to make sure that while the total budget is increased there will be no budget decline in each park for each of the two fire programs. The gradient approach will share this same budgeting principle; however, it also allocates new budget to each park in each program proportionally according to the 
marginal investment return from each program in each park. Budget allocation using the gradient approach is directly related with the current program performance in each park for each fire program. Because the gradient method can only guarantee the local optimality, the budget allocation based on it may not be globally optimal.

\section{REFERENCES}

[1] King, K.J., Bradstock, R.A., Cary, G.J., Chapman, J. \& Marsden-Smedley, J.B., The relative importance of fine-scale fuel mosaics on reducing fire risk in south-west Tasmania, Australia. International Journal of Wildland Fire, 17, pp. 421-430, 2008. http://dx.doi.org/10.1071/WF07052

[2] Reinhardt, E.D., Keane, R.E., Calkin, D.E. \& Cohen, J.D., Objectives and considerations for wildland fuel treatment in forested ecosystems of the interior western United States. Forest Ecology and Management, 256, pp. 1997-2006, 2008. http://dx.doi.org/10.1016/j.foreco.2008.09.016

[3] Minas, J., Hearne, J. \& Martell, D., An integrated optimization model for fuel management and fire suppression preparedness planning. Annals of Operations Research, 232(1), pp. 201-215, 2015.

[4] United States Office of Management and Budget, The budget for fiscal year 2015, p. 218, Retrieved from http://www.whitehouse.gov/sites/default/files/omb/budget/ fy2015/assets/budget.pdf

[5] United States Congress, FLAME Act of 2009, H.R. 2996. In One Hundred Eleventh Congress of the United States of America, 2009.

[6] United States Congress, Healthy forests restoration act of 2003, H.R. In 1904, One Hundred Eigth Congress of the United States of America, 2003.

[7] National Wildfire Coordinating Group, Wildland fire incident management field guide, Retrieved from http://www.nifc.gov/nicc/logistics/references/Wildland\%20Fire\%20 Incident\%20Management\%20Field\%20Guide.pdf

[8] Martell, D., A review of operational research studies in forest fire management. Canadian Journal of Forest Research, 12(2), pp. 119-140, 1982. http://dx.doi.org/10.1139/x82-020

[9] Rideout, D.B., Rossi, D. \& Kernohan, N., Economics of ecosystem restoration: using derived demand to promote sustainable development. In Ecosystems and Sustainable Development X, eds. J.L. Miralles Garcia \& C.A. Brebbia, WIT Press: Southhampton, $\mathrm{UK}, 2015$. 